\title{
Culture Establishment of Coccinia grandis (L.) through Nodal Segments under in vitro Conditions
}

\author{
Mohammad Amin ${ }^{1 *}$, A. V. D. Dorajee Rao ${ }^{2}$, K. Ravindra Kumar ${ }^{3}$ \\ and D. R. Salomi Suneetha ${ }^{4}$ \\ ${ }^{1}$ Department of Vegetable Science, ${ }^{2,3}$ Department of Horticulture, ${ }^{4}$ Department of \\ Biochemistry, College of Horticulture, Dr. Y. S. R Horticultural University, \\ Venkataramannagudem, West Godavari, India \\ *Corresponding author
}

A B S T R A C T

\begin{tabular}{|l|}
\hline K e y w o r d s \\
Coccinia, Nodal \\
segments, Culture \\
establishment, \\
Shoot proliferation, \\
In vitro
\end{tabular}

An experiment was conducted on culture establishment of Coccinia grandis (L.) through nodal segments under in vitro conditions with an objective to standardise a viable protocol for in vitro propagation. Significant results were observed for number of buds sprouted, percent of shoot bud induction, number of days taken for shoot initiation, number of shoots per explant, shoot length, number of leaves per shoot and vitrification For shoot induction and succeeding growth was effectively induced by TDZ at lower $\left(0.5 \mathrm{mg} \mathrm{l}^{-1}\right)$ concentration as compared to higher $\left(1.0 \mathrm{mg}^{-1}\right)$ concentration). For shoot proliferation $3.0 \mathrm{mg} \mathrm{l}^{-1} \mathrm{BAP}+0.25 \mathrm{mg} \mathrm{l}^{-1} \mathrm{NAA}\left(\mathrm{T}_{4}\right)$ and $4.0 \mathrm{mg}$ $1^{-1} \mathrm{BAP}+0.25 \mathrm{mg}^{-1} \mathrm{NAA}\left(\mathrm{T}_{5}\right)$ in MS medium were more efficient for inducing maximum number of micro shoots and leaves.

\section{Introduction}

Coccinia grandis L. is commonly known as the ivy gourd, also known as scarlet gourd, tindora, and kowai fruit is a tropical vine is a slender dioecious perennial climber with tuberous roots; belongs to the family Cucurbitaceae and is described as 'Indian substitute for Insulin". It grows widely throughout India and other tropical countries but it is commonly found in the southern Indian states, where it forms a part of the local cuisine. Ivy gourd has been extensively used in ayurvedic and unani practices in the Indian Subcontinent. The plant is known to have antidiabetic, anti inflammatory, antipyretic, analgesic, antispasmodic, antimicrobial, anthelmintic, cathartic and expectorant activities. It contains several phytoconstituents such as cephalandrol, iritriacontane, Triterpenoids, alkaloids and tannins. The plant also possesses hypoglycemic effects and acts as insulin mimetic (Thiripurasundari and Rao, 2012). 
Problems associated with its cultivation include the shortage of seedling material from cuttings of mature stems and viral diseases that may lead to scarcity of quality and disease free plant material. General barriers like poor seed setting and low seed germination is a common in Cucurbits including Coccinea, probably due to the presence of a thin nucellar membrane lending impermeability to water and gases making them dormant for many days (Thiripurasundari and Rao, 2012). The use of tissue culture techniques for clonal propagation has become the most widely used application of tissue culture technology in horticultural crops in the recent years (Thorpe, 1990).

In vitro propagation can effectively escape from these problems and as such micro propagated material are reported to grow faster and mature earlier than seed propagated ones (Vasil and Vasil, 1980).

The present investigation is undertaken with a view to standardise the techniques for culture establishment of in vitro micro propagated plantlets; aimed at developing a viable protocol for propagation of coccinea through in vitro procedures.

\section{Materials and Methods}

The present study was conducted at Tissue Culture Laboratory, Horticultural Research station, Kovvur, College of Horticulture, Dr. Y. S. R. Horticultural University, Venkataramannagudem, Andhra Pradesh, during the year 2019-2020.

\section{Plant material}

Nodal segments of ivy gourd were involved in the present study. Nodal segments (two cm long) were collected from young vines maintained in the propagation chamber at the
HRS, Kovvur and were used for the preparation of explants.

The nodal segments were pre-treated with Carbandazim (0.2\%) + Mancozeb \& Metalaxyl $(0.2 \%)+200$ ppm 8 HQ for $60 \mathrm{~min}$ and sterilized with $0.1 \% \mathrm{HgCl}_{2}$ for 4 minutes for better culture establishment and shoot proliferation.

\section{Culture media}

\section{Preparation of culture media}

Stock solutions of macro and micro elements and organic constituents

Stock solutions of macro and micro elements were prepared by dissolving appropriate quantities of each element in accordance with MS medium. For stock solution of ferrous sulphate $\left(\mathrm{FeSO}_{4}\right)$, required quantities of $\mathrm{FeSO}_{4} \cdot 7 \mathrm{H}_{2} \mathrm{O}$ and $\mathrm{Na}_{2}$ EDTA were dissolved separately; the $\mathrm{Na}_{2}$ EDTA solution was slightly warmed in order to aid the completion of the chelation reaction and was added gently to the $\mathrm{FeSO}_{4}$ solution. The final volume was made up with distilled water. Stock solutions of the organic constituents namely the vitamins and amino acids were prepared and maintained separately.

\section{Auxins}

NAA and IBA: Hundred mg of auxin was dissolved in 2 to $3 \mathrm{ml}$ of $1 \mathrm{~N} \mathrm{NaOH}$, heated slightly and the final volume was made up to $100 \mathrm{ml}$ with sterile double distilled water.

\section{Cytokinin}

BAP: A quantity of $100 \mathrm{mg}$ of BAP was dissolved in 2 to $3 \mathrm{ml}$ of $1 \mathrm{~N} \mathrm{HCl}$, heated slightly and the final volume was made up to $100 \mathrm{ml}$ with sterile double distilled water. 


\section{Gibberellin}

$\mathrm{GA}_{3}$ : Hundred $\mathrm{mg}$ of $\mathrm{GA}_{3}$ was dissolved in distilled water and the final volume was made up to $100 \mathrm{ml}$ and $\mathrm{pH}$ was adjusted to 5.7. The prepared stocks were stored in refrigerator at $4-5^{0} \mathrm{C}$.

\section{Results and Discussion}

\section{In vitro culture establishment}

The experiment was aimed at standardising growth regulator concentrations for in vitro culture establishment and proliferation. The results for number of buds sprouted, percent of shoot bud induction, number of days taken for shoot initiation, number of shoots per explant, shoot length, number of leaves per shoot and vitrification are described hereunder.

\section{Number of buds sprouted}

The data revealed that there were significant differences among the treatments. The mean data for number of buds sprouted was (6.25) (Table 3.1). Among the treatments, the maximum number of buds sprouted was achieved by $\mathrm{MS}+3.0 \mathrm{mg} \mathrm{l}^{-1} \mathrm{BAP}+0.1 \mathrm{mg} \mathrm{l}^{-1}$ NAA $\left(\mathrm{T}_{4}\right)(9.50)$ which was on par with MS + $0.5 \mathrm{mg} \mathrm{l}^{-1} \mathrm{TDZ}\left(\mathrm{T}_{5}\right)(9.25), \mathrm{MS}+2.0 \mathrm{mg} \mathrm{l}^{-1}$ $\mathrm{BAP}+0.1 \mathrm{mg} \mathrm{l}^{-1} \mathrm{NAA}\left(\mathrm{T}_{3}\right)(6.75)$ and $\mathrm{MS}+$ $1.0 \mathrm{mg} \mathrm{l}^{-1} \mathrm{TDZ}\left(\mathrm{T}_{6}\right)(6.25)$ at $1 \%$ level of probability. However, the minimum number of buds sprouted with MS medium devoid of any growth regulators as Control $\left(\mathrm{T}_{7}\right)(1.75)$.

\section{Percent of shoot bud induction (\%)}

There were significant differences among the treatments with respect to per cent of shoot bud induction. The mean data for per cent of buds sprouted was $(62.50 \%)$ (Table 3.2$)$. The maximum per cent of buds sprouted was achieved by $\mathrm{MS}+3.0 \mathrm{mg} \mathrm{l}^{-1} \mathrm{BAP}+0.1 \mathrm{mg} \mathrm{l}^{-1}$
NAA $\left(\mathrm{T}_{4}\right)(95.00 \%)$ which was on par with $\mathrm{MS}+0.5 \mathrm{mg} \mathrm{l}^{-1} \mathrm{TDZ}\left(\mathrm{T}_{5}\right)(92.5 \%), \mathrm{MS}+2.0$ $\mathrm{mg} \mathrm{l}^{-1} \mathrm{BAP}+0.1 \mathrm{mg} \mathrm{l}^{-1} \mathrm{NAA}\left(\mathrm{T}_{3}\right)(67.50 \%)$ and $\mathrm{MS}+1.0 \mathrm{mg} \mathrm{l}^{-1} \mathrm{TDZ}\left(\mathrm{T}_{6}\right)(62.50 \%)$ at $1 \%$ level of probability. The minimum number of buds sprouted with MS medium devoid of any growth regulators as control $\left(\mathrm{T}_{7}\right)(17.50 \%)$.

\section{Number of shoots per explant}

The data revealed that there were significant differences among the treatments. The mean data for number of shoots per explant was (1.33) (Table 3.2). The maximum number of shoots per explant was recorded by MS + 3.0 $\mathrm{mg}^{-1} \mathrm{BAP}+0.1 \mathrm{mg} \mathrm{l}^{-1} \mathrm{NAA}\left(\mathrm{T}_{4}\right)(2.25)$. The minimum number of shoots per explant (1.00) was recorded by $\mathrm{MS}+0.5 \mathrm{mg}^{-1} \mathrm{BAP}+0.1$ $\mathrm{mg}^{-1} \mathrm{NAA}\left(\mathrm{T}_{1}\right)$ (1.00) which was on par with $\mathrm{MS}+1.0 \mathrm{mg} \mathrm{l}^{-1} \mathrm{BAP}+0.1 \mathrm{mg} \mathrm{l}^{-1} \mathrm{NAA}$ $\left(\mathrm{T}_{2}\right)(1.00), \mathrm{MS}+2.0 \mathrm{mg} \mathrm{l}^{-1} \mathrm{BAP}+0.1 \mathrm{mg} \mathrm{l}^{-1}$ NAA $\left(\mathrm{T}_{3}\right)(1.25), \mathrm{MS}+0.5 \mathrm{mg} \mathrm{l}^{-1} \mathrm{TDZ}\left(\mathrm{T}_{5}\right)$ and $\mathrm{MS}+1.0 \mathrm{mg} \mathrm{l}^{-1} \mathrm{TDZ}\left(\mathrm{T}_{6}\right)(1.50)$ at $1 \%$ level of probability.

However, minimum number of shoots per explant was recorded by MS medium devoid of any growth regulators as control $\left(\mathrm{T}_{7}\right)$ (1.00).

\section{Culture Establishment Index (CEI)}

Culture Establishment Index (CEI) was found to vary significantly among the treatments. The mean CEI value was 83.9 (Table 3.2). The maximum number of shoots per explant was recorded by $\mathrm{MS}+3.0 \mathrm{mg} \mathrm{l}^{-1} \mathrm{BAP}+0.1$ $\mathrm{mg}^{-1}$ NAA $\left(\mathrm{T}_{4}\right)$ (213.8). The minimum number of shoots per explant (1.00) was recorded by $\mathrm{MS}+0.5 \mathrm{mg} \mathrm{l}^{-1} \mathrm{BAP}+0.1 \mathrm{mg} \mathrm{l}^{-1}$ NAA $\left(\mathrm{T}_{1}\right)$ (1.00) which was significantly superior to $\mathrm{MS}+1.0 \mathrm{mg} \mathrm{l}^{-1} \mathrm{TDZ}\left(\mathrm{T}_{6}\right)(138.8)$ at $1 \%$ level of probability. However, minimum culture establishment index was recorded by $\mathrm{MS}+0.5 \mathrm{mg} \mathrm{l}^{-1} \mathrm{TDZ}\left(\mathrm{T}_{5}\right)(17.5)$. 


\section{Number of days taken for shoot initiation}

Significant differences were observed among the treatments. The mean data for number of days taken for shoot initiation was (11.08) (Table 3.3). The minimum number of days taken for shoot initiation was achieved by MS $+3.0 \mathrm{mg} / \mathrm{l} \mathrm{BAP}+0.1 \mathrm{mg} \mathrm{l}^{-1} \mathrm{NAA}\left(\mathrm{T}_{4}\right)(7.25)$ which was on par with MS $+1.0 \mathrm{mg} \mathrm{l}^{-1} \mathrm{TDZ}$ $\left(\mathrm{T}_{6}\right)(8.25)$ and $\mathrm{MS}+2.0 \mathrm{mg} \mathrm{l}^{-1} \mathrm{BAP}+0.1$ mg $1^{-1}$ NAA $\left(\mathrm{T}_{3}\right)(10.00)$ but significantly superior to the MS $+1.0 \mathrm{mg} \mathrm{l}^{-1} \mathrm{BAP}+0.1 \mathrm{mg}$ $1^{-1}$ NAA $\left(T_{2}\right)(12.75)$. However, the maximum number of days taken for shoot initiation was observed in the MS medium devoid of any growth regulators as $\mathrm{MS}+0.5 \mathrm{mg} \mathrm{l}^{-1} \mathrm{TDZ}$ $\left(\mathrm{T}_{6}\right)\left(\mathrm{T}_{5}\right)(17.50)$.

\section{Shoot length $(\mathrm{cm}) \mathbf{1 5}$ days after inoculation}

Significant differences were recorded among the treatments. The mean shoot length was $(1.55 \mathrm{~cm})$ (Table 3.3). The maximum shoot length was noticed in MS + 3.0 mg $\mathrm{l}^{-1} \mathrm{BAP}+$ $0.1 \mathrm{mg} \mathrm{l}^{-1} \mathrm{NAA}\left(\mathrm{T}_{4}\right)(3.20 \mathrm{~cm})$ followed by $\mathrm{MS}+2.0 \mathrm{mg} \mathrm{l}^{-1} \mathrm{BAP}+0.1 \mathrm{mg} \mathrm{l}^{-1} \mathrm{NAA}\left(\mathrm{T}_{3}\right)$ $(1.75 \mathrm{~cm})$ but significantly superior to the MS $+1.0 \mathrm{mg} \mathrm{l}^{-1} \mathrm{TDZ}\left(\mathrm{T}_{6}\right)$ (1.65). However, the minimum shoot length was recorded in MS medium devoid of any growth regulators as $\mathrm{MS}+0.5 \mathrm{mg} \mathrm{l}^{-1} \mathrm{TDZ}\left(\mathrm{T}_{5}\right)(0.47 \mathrm{~cm})$.

Similar to the present study, maximum shoot bud induction in cucumber, was noticed at 3.0 $\mathrm{mg} \mathrm{l}^{-1} \mathrm{BAP}$ in comparison to $\mathrm{Kn}$ along with low levels of auxin $\left(0.5 \mathrm{mg} \mathrm{l}^{-1}\right)$ in MS medium (Ugandhar et al., 2015).

Aragaw and Abebe (2017) reported that medium with high inorganic nutrient salt and optimum level of growth regulator performed the best for initiation of maximum number of shoots in green pepper. The shoot tip explants produced maximum multiple shoots at optimum level of cytokine growth regulator and survival percentage (Mukund, 1998) in banana.
Hasan (2019) showed that $2 \mathrm{mg} \mathrm{l}^{-1} \mathrm{BAP}$ with $0.5 \mathrm{mg} \mathrm{l}^{-1}$ NAA induced multiple shoots from Citrus jambhiri. Shekhawat et al., (2014) achieved maximum number of shoot regeneration on MS medium supplemented with $1.0 \mathrm{mg} \mathrm{l}^{-1}$ each of BAP and $\mathrm{Kn}$ in Coccinia indica. Aasim et al., (2008) also recorded the maximum number and length of shoots per explant of cowpea on MS containing $0.5 \mathrm{mg} \mathrm{l}^{-1}$ BAP with $0.1 \mathrm{mg} \mathrm{l}^{-1}$ NAA.

Literature suggests that BAP is more effective at combinations of $1.0 \mathrm{mg} / \mathrm{L}$ to $3.0 \mathrm{mg} / \mathrm{L}$ in many plant systems (Senthilkumar et al., 2007; Arulanandam and Ghanthikumar 2011). The same has been confirmed in the present study where the shoot induction in multiple numbers and maximum growth of shoots was exhibited by $3.0 \mathrm{mg} \mathrm{l}^{-1} \mathrm{BAP}+0.1 \mathrm{mg} \mathrm{l}^{-1}$ NAA $\left(\mathrm{T}_{4}\right)$ followed by $2.0 \mathrm{mg} \mathrm{l}^{-1} \mathrm{BAP}+0.1$ $\mathrm{mg} \mathrm{l}^{-1}$ NAA $\left(\mathrm{T}_{3}\right)$ in MS medium.

As regards to the performance of TDZ for shoot induction, Kahia et al., (2016) reported that the optimum TDZ concentration was found to be $0.025 \mathrm{mM}$. Increasing the concentration beyond the optimum led to significant reduction of the mean number of microshoots. This trend of decreased efficiency beyond the optimum TDZ concentration was also observed in somatic embryogenesis of Phalaenopsis aphrodite (Feng and Chen, 2014) and in micropropagation of lentils (Khawar et al., 2004).

In the present study shoot induction and succeeding growth was effectively induced by TDZ at lower $\left(0.5 \mathrm{mg} \mathrm{l}^{-1}\right)$ concentration as compared to higher $\left(\begin{array}{llll}1.0 & \mathrm{mg} & \mathrm{l}^{-1}\end{array}\right)$ concentration). A possible explanation for this could be due to the fact that it is stable and biologically active at lower concentrations (Mok and Mok, 1987). 
Similar observations were made in woody plant species, where low levels of TDZ have been known to induce the axillary shoot proliferation but higher levels may inhibit it and rather promote callus formation (Huetteman, 1993).

\section{Number of leaves per shoot}

The results on number of leaves per shoot revealed significant differences among the treatments. The mean number of leaves per shoot was (3.21) (Table 3.3). The maximum number of leaves per shoot was recorded by $\mathrm{MS}+3.0 \mathrm{mg} \mathrm{l}^{-1} \mathrm{BAP}+0.1 \mathrm{mg} \mathrm{l}^{-1} \mathrm{NAA}\left(\mathrm{T}_{4}\right)$ (5.0) followed by $\mathrm{MS}+1.0 \mathrm{mg} \mathrm{l}^{-1} \mathrm{TDZ}\left(\mathrm{T}_{6}\right)$ (3.50) but significantly superior to the MS + $2.0 \mathrm{mg} \mathrm{l}^{-1} \mathrm{BAP}+0.1 \mathrm{mg} \mathrm{l}^{-1} \mathrm{NAA}\left(\mathrm{T}_{3}\right)(3.25$ $\mathrm{cm})$. The minimum number of leaves per shoot was recorded in MS medium devoid of any growth regulators as $\mathrm{MS}+0.5 \mathrm{mg} \mathrm{l}^{-1}$
$\operatorname{TDZ}\left(\mathrm{T}_{5}\right)(2.25)$

The meritorious results as recorded by the addition of $3.0 \mathrm{mg} \mathrm{l}^{-1} \mathrm{BAP}+0.1 \mathrm{mg} \mathrm{l}^{-1} \mathrm{NAA}$ $\left(\mathrm{T}_{4}\right)$ and $0.5 \mathrm{mg} \mathrm{l}^{-1} \mathrm{TDZ}\left(\mathrm{T}_{5}\right)$ were also extended to the leaf induction, which indicates that the differentiation of aerial plant system was effectively achieved by these chemicals in MS medium.

This signifies the fact that the regeneration medium with sufficient level of inorganic mineral salts as in MS medium and optimum level of cytokinin growth regulator as in case of $3 \mathrm{mg} \mathrm{l}^{-1}$ of BAP $+0.1 \mathrm{mg} \mathrm{l}^{-1}$ of NAA or 0.5 $\mathrm{mg} \mathrm{l}^{-1}$ of TDZ (Ugandhar et al., (2015) and Aragaw and Abebe (2017)) could be adopted in standard protocols for in vitro propagation of coccinea nodal segments at shoot initiation phase.

Table.3.1 Per cent bud sprouting as influenced different growth regulator concentrations for in vitro culture establishment of coccinea nodal segments

\begin{tabular}{|c|c|c|}
\hline Treatments Details & $\begin{array}{l}\text { No. of buds } \\
\text { cultured }\end{array}$ & $\begin{array}{l}\text { No. of buds } \\
\text { sprouted }\end{array}$ \\
\hline $\begin{array}{c}\mathrm{T}_{1}: \mathrm{MS}+0.5 \mathrm{mg} \mathrm{l}^{-1} \mathrm{BAP}+0.1 \\
\mathrm{mg} \mathrm{l}^{-1} \mathrm{NAA}\end{array}$ & 10 & $3.00(1.73)$ \\
\hline $\begin{aligned} \mathrm{T}_{2}: & \mathrm{MS}+1.0 \mathrm{mg} \mathrm{l}^{-1} \mathrm{BAP}+0.1 \\
\mathrm{mg} \mathrm{l}^{-1} \mathrm{NAA} & \end{aligned}$ & 10 & $4.00(2.00)$ \\
\hline $\begin{array}{c}\mathrm{T}_{3}: \mathrm{MS}+2.0 \mathrm{mg} \mathrm{l}^{-1} \mathrm{BAP}+0.1 \\
\mathrm{mg} \mathrm{l}^{-1} \mathrm{NAA}\end{array}$ & 10 & $6.75(2.60)$ \\
\hline $\begin{aligned} \mathrm{T}_{4}: & \mathrm{MS}+3.0 \mathrm{mg} \mathrm{l}^{-1} \mathrm{BAP}+0.1 \\
\mathrm{mg} \mathrm{l}^{-1} \mathrm{NAA} & \end{aligned}$ & 10 & $9.50(3.08)$ \\
\hline $\mathrm{T}_{5}: \mathrm{MS}+0.5 \mathrm{mg} \mathrm{l}^{-1} \mathrm{TDZ}$ & 10 & $1.75(1.32)$ \\
\hline$T_{6}: M S+1.0 \mathrm{mg} \mathrm{l}^{-1} \mathrm{TDZ}$ & 10 & $9.25(3.04)$ \\
\hline $\begin{array}{l}T_{7}: \text { MS medium devoid of any } \\
\text { growth regulators as Control }\end{array}$ & 10 & $6.25(2.50)$ \\
\hline Mean & 10.00 & $6.25(2.08)$ \\
\hline S Em & & 0.86 \\
\hline CD at 0.01 & & 3.66 \\
\hline CD at 0.05 & & 2.62 \\
\hline
\end{tabular}


Table.3.2 Per cent shoot bud induction and Culture Establishment Index as influenced different growth regulator concentrations for in vitro culture establishment of coccinea nodal segments

\begin{tabular}{|c|c|c|c|}
\hline Treatments Details & \% shoot bud induction & No. of shoots per explant & $\begin{array}{l}\text { Culture Establishment } \\
\text { Index (CEI) }\end{array}$ \\
\hline $\begin{aligned} & \mathrm{T}_{1}: \mathrm{MS}+0.5 \mathrm{mg} \mathrm{l}^{-1} \mathrm{BAP}+0.1 \mathrm{mg} \mathrm{l}^{-1} \\
& \text { NAA }\end{aligned}$ & $\begin{array}{c}30.00 \\
(33.20)\end{array}$ & $1.00(1.00)$ & 30.0 \\
\hline $\begin{aligned} \mathrm{T}_{2}: & \mathrm{MS}+1.0 \mathrm{mg} \mathrm{l}^{-1} \mathrm{BAP}+0.1 \mathrm{mg} \mathrm{l}^{-1} \\
\text { NAA } & \end{aligned}$ & $\begin{array}{c}40.00 \\
(39.22)\end{array}$ & $1.00(1.00)$ & 40.0 \\
\hline $\begin{aligned} \mathrm{T}_{3}: & \mathrm{MS}+2.0 \mathrm{mg} \mathrm{l}^{-1} \mathrm{BAP}+0.1 \mathrm{mg} \mathrm{l}^{-1} \\
& \text { NAA }\end{aligned}$ & $\begin{array}{c}67.50 \\
(55.22)\end{array}$ & $1.25(1.12)$ & 84.4 \\
\hline $\begin{aligned} \mathrm{T}_{4}: & \mathrm{MS}+3.0 \mathrm{mg} \mathrm{l}^{-1} \mathrm{BAP}+0.1 \mathrm{mg} \mathrm{l}^{-1} \\
& \mathrm{NAA}\end{aligned}$ & $\begin{array}{c}95.00 \\
(77.05)\end{array}$ & $2.25(1.50)$ & 213.8 \\
\hline $\mathrm{T}_{5}: \mathrm{MS}+0.5 \mathrm{mg} \mathrm{l}^{-1} \mathrm{TDZ}$ & $\begin{array}{l}17.50 \\
(24.72)\end{array}$ & $1.00(1.00)$ & 17.5 \\
\hline $\mathrm{T}_{6}: \mathrm{MS}+1.0 \mathrm{mg} \mathrm{l}^{-1} \mathrm{TDZ}$ & $\begin{array}{c}92.50 \\
(74.08)\end{array}$ & $1.50(1.22)$ & 138.8 \\
\hline $\begin{aligned} \mathrm{T}_{7}: & \text { MS medium devoid of any } \\
& \text { growth regulators as Control }\end{aligned}$ & $\begin{array}{c}62.50 \\
(52.22)\end{array}$ & $1.00(1.00)$ & 62.5 \\
\hline Mean & $\begin{array}{c}\text { 62.50 } \\
(46.07)\end{array}$ & $1.33(0.98)$ & 83.86 \\
\hline S Em & 7.35 & 0.10 & 14.04 \\
\hline CD at 0.01 & 31.32 & 0.42 & 59.83 \\
\hline CD at 0.05 & 22.46 & 0.30 & 42.91 \\
\hline
\end{tabular}

Table.3.3 Days taken for shoot initiation and shoot parameters as influenced by different growth regulator concentrations for in vitro culture establishment of coccinea nodal segments

\begin{tabular}{|c|c|c|c|}
\hline Treatments Details & $\begin{array}{l}\text { No. of days taken for } \\
\text { shoot initiation }\end{array}$ & $\begin{array}{c}\text { Shoot length }(\mathrm{cm}) 15 \text { days } \\
\text { after inoculation }\end{array}$ & $\begin{array}{c}\text { No. of leaves per } \\
\text { shoot }\end{array}$ \\
\hline $\begin{aligned} \mathrm{T}_{1}: & \mathrm{MS}+0.5 \mathrm{mg} \mathrm{l}^{-1} \mathrm{BAP}+ \\
& 0.1 \mathrm{mg} \mathrm{l}^{-1} \mathrm{NAA}\end{aligned}$ & 14.25 & 0.90 & 2.75 \\
\hline $\begin{aligned} \mathrm{T}_{2}: & \mathrm{MS}+1.0 \mathrm{mg} \mathrm{l}^{-1} \mathrm{BAP}+0.1 \\
& \mathrm{mg} \mathrm{l}^{-1} \mathrm{NAA}\end{aligned}$ & 12.75 & 1.07 & 2.50 \\
\hline $\begin{aligned} \mathrm{T}_{3}: & \mathrm{MS}+2.0 \mathrm{mg} \mathrm{l}^{-1} \mathrm{BAP}+0.1 \\
& \mathrm{mg} \mathrm{l}^{-1} \mathrm{NAA}\end{aligned}$ & 10.00 & 1.75 & 3.25 \\
\hline $\begin{aligned} \mathrm{T}_{4}: & \mathrm{MS}+3.0 \mathrm{mg} \mathrm{l}^{-1} \mathrm{BAP}+0.1 \\
& \mathrm{mg} \mathrm{l}^{-1} \mathrm{NAA}\end{aligned}$ & 7.25 & 3.20 & 5.00 \\
\hline $\mathrm{T}_{5}: \mathrm{MS}+0.5 \mathrm{mg} \mathrm{l}^{-1} \mathrm{TDZ}$ & 17.50 & 0.47 & 2.25 \\
\hline $\mathrm{T}_{6}: \mathrm{MS}+1.0 \mathrm{mg} \mathrm{l}^{-1} \mathrm{TDZ}$ & 8.25 & 1.65 & 3.50 \\
\hline $\begin{aligned} \mathrm{T}_{7}: & \text { MS medium devoid of } \\
& \text { any growth regulators as } \\
& \text { Control. }\end{aligned}$ & 10.75 & 1.12 & 2.75 \\
\hline Mean & 11.08 & 1.55 & 3.21 \\
\hline S Em & 0.74 & 0.19 & 0.20 \\
\hline CD at 0.01 & 3.14 & 0.79 & 0.85 \\
\hline CD at 0.05 & 2.25 & 0.57 & 0.61 \\
\hline
\end{tabular}


Table.3.4 Shoot parameters as influenced by different growth regulator concentrations during in vitro culture proliferation of coccinea nodal segments

\begin{tabular}{|c|c|c|c|}
\hline Treatments Details & $\begin{array}{l}\text { No. of buds } \\
\text { cultured }\end{array}$ & $\begin{array}{l}\text { No. of shoots per each } \\
\text { micro shoot } 30 \text { days } \\
\text { after inoculation }\end{array}$ & $\begin{array}{c}\text { Shoot length }(\mathrm{cm}) \text { at } \\
30 \text { days after } \\
\text { inoculation }\end{array}$ \\
\hline $\mathrm{T}_{1}: \mathrm{MS}+0.5 \mathrm{mg} \mathrm{l}^{-1} \mathrm{BAP}+0.25 \mathrm{mg} \mathrm{l}^{-1} \mathrm{NAA}$ & 10 & 1.50 & 3.65 \\
\hline $\mathrm{T}_{2}: \mathrm{MS}+1.0 \mathrm{mg} \mathrm{l}^{-1} \mathrm{BAP}+0.25 \mathrm{mg} \mathrm{l}^{-1} \mathrm{NAA}$ & 10 & 2.50 & 3.40 \\
\hline $\mathrm{T}_{3}: \mathrm{MS}+2.0 \mathrm{mg} \mathrm{l}^{-1} \mathrm{BAP}+0.25 \mathrm{mg} \mathrm{l}^{-1} \mathrm{NAA}$ & 10 & 3.50 & 3. 00 \\
\hline $\mathrm{T}_{4}: \mathrm{MS}+3.0 \mathrm{mg} \mathrm{l}^{-1} \mathrm{BAP}+0.25 \mathrm{mg} \mathrm{l}^{-1} \mathrm{NAA}$ & 10 & 7.50 & 2.60 \\
\hline $\mathrm{T}_{5}: \mathrm{MS}+4.0 \mathrm{mg} \mathrm{l}^{-1} \mathrm{BAP}+0.25 \mathrm{mg} \mathrm{l}^{-1} \mathrm{NAA}$ & 10 & 5.50 & 1.75 \\
\hline $\mathrm{T}_{6}: \mathrm{MS}+5.0 \mathrm{mg} \mathrm{l}^{-1} \mathrm{BAP}+0.25 \mathrm{mg} \mathrm{l}^{-1} \mathrm{NAA}$ & 10 & 2.50 & 1.45 \\
\hline $\begin{array}{l}\mathrm{T}_{7}: \text { MS medium devoid of any growth } \\
\text { regulators as Control }\end{array}$ & 10 & 1.00 & 2.30 \\
\hline Mean & $\mathbf{1 0 . 0 0}$ & 3.43 & 2.59 \\
\hline S Em & & 0.66 & 0.16 \\
\hline CD at 0.01 & & 2.82 & 0.70 \\
\hline CD at 0.05 & & 2.02 & 0.50 \\
\hline
\end{tabular}

Table.3.5 Leaf parameters as influenced by different growth regulator concentrations during in vitro culture proliferation of coccinea nodal segments

\begin{tabular}{|c|c|c|c|}
\hline Treatments Details & $\begin{array}{l}\text { No. of leaves per } \\
\text { shoot }\end{array}$ & $\begin{array}{l}\text { Leaf area }\left(\mathrm{cm}^{2}\right) \\
\left(3^{\text {rd }} \text { leaf from top }\right)\end{array}$ & $\begin{array}{l}\text { Vitrification } \\
\quad(\%)\end{array}$ \\
\hline $\mathrm{T}_{1}: \mathrm{MS}+0.5 \mathrm{mg} \mathrm{l}^{-1} \mathrm{BAP}+0.25 \mathrm{mg} \mathrm{l}^{-1} \mathrm{NAA}$ & 3.00 & 6.2 & $\begin{array}{c}0.00 \\
(0.71)\end{array}$ \\
\hline $\mathrm{T}_{2}: \mathrm{MS}+1.0 \mathrm{mg} \mathrm{l}^{-1} \mathrm{BAP}+0.25 \mathrm{mg} \mathrm{l}^{-1} \mathrm{NAA}$ & 5.00 & 5.6 & $\begin{array}{c}0.00 \\
(0.71)\end{array}$ \\
\hline $\mathrm{T}_{3}: \mathrm{MS}+2.0 \mathrm{mg} \mathrm{l}^{-1} \mathrm{BAP}+0.25 \mathrm{mg} \mathrm{l}^{-1} \mathrm{NAA}$ & 8.00 & 3.8 & $\begin{array}{c}0.25 \\
(0.87)\end{array}$ \\
\hline $\mathrm{T}_{4}: \mathrm{MS}+3.0 \mathrm{mg} \mathrm{l}^{-1} \mathrm{BAP}+0.25 \mathrm{mg} \mathrm{l}^{-1} \mathrm{NAA}$ & 13.00 & 2.1 & $\begin{array}{c}1.00 \\
(1.22)\end{array}$ \\
\hline $\mathrm{T}_{5}: \mathrm{MS}+4.0 \mathrm{mg} \mathrm{l}^{-1} \mathrm{BAP}+0.25 \mathrm{mg} \mathrm{l}^{-1} \mathrm{NAA}$ & 7.00 & 5.0 & $\begin{array}{c}3.62 \\
(2.03)\end{array}$ \\
\hline $\mathrm{T}_{6}: \mathrm{MS}+5.0 \mathrm{mg} \mathrm{l}^{-1} \mathrm{BAP}+0.25 \mathrm{mg} \mathrm{l}^{-1} \mathrm{NAA}$ & 6.00 & 3.8 & $\begin{array}{l}10.25 \\
(3.28)\end{array}$ \\
\hline $\begin{aligned} & \mathrm{T}_{7}: \text { MS medium devoid of any growth } \\
& \text { regulators as Control }\end{aligned}$ & 2.00 & 3.2 & $\begin{array}{c}0.00 \\
(0.71)\end{array}$ \\
\hline Mean & 6.29 & 4.24 & $\begin{array}{c}\mathbf{2 . 1 6} \\
(1.36)\end{array}$ \\
\hline S Em & 0.73 & 0.29 & 0.28 \\
\hline CD at 0.01 & 3.10 & 1.22 & 1.18 \\
\hline CD at 0.05 & 2.22 & 0.88 & 0.85 \\
\hline
\end{tabular}


Fig.3.1 Shoot proliferation of coccinea nodal segments in vitro as influenced by different growth regulator concentrations
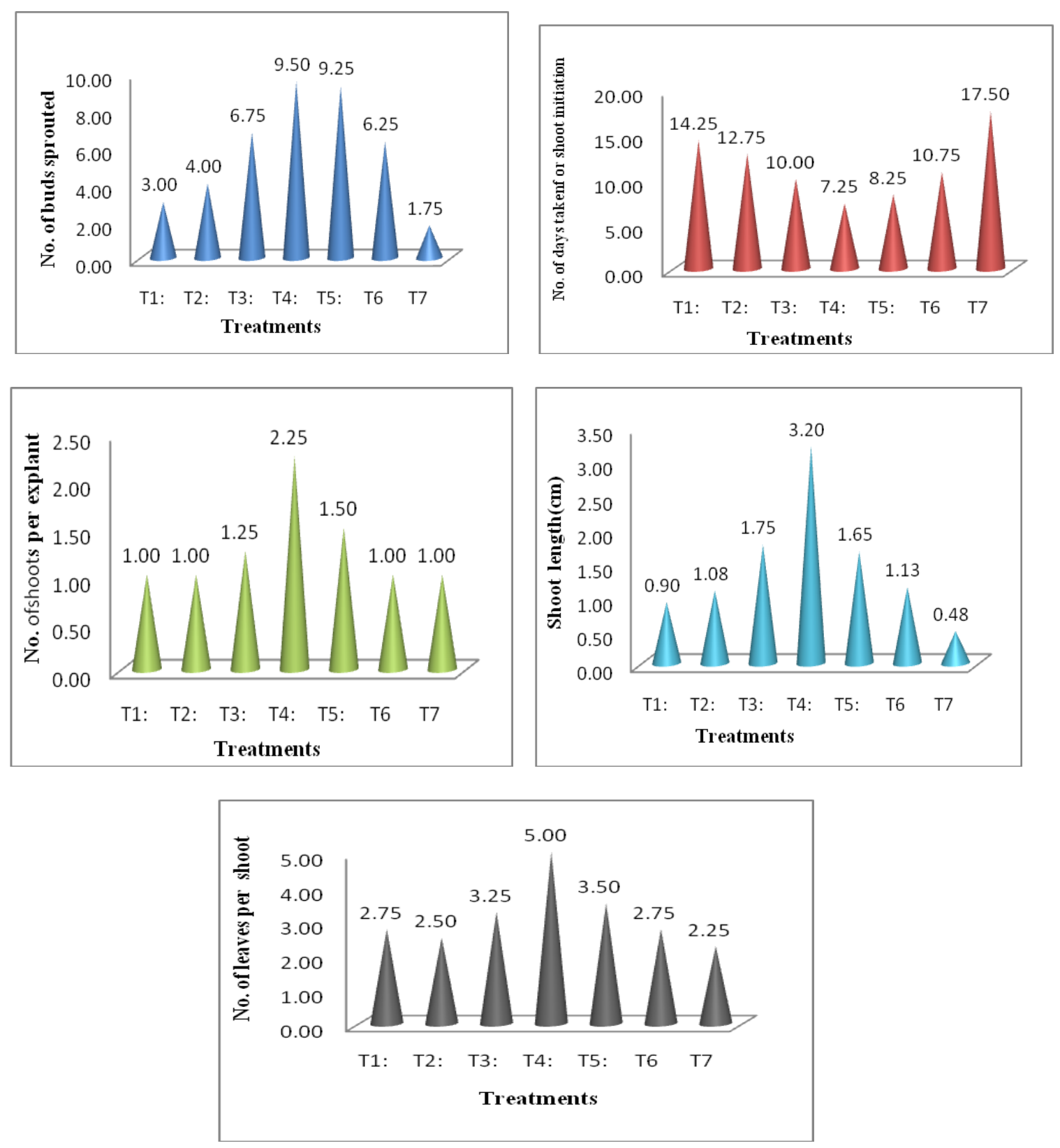

Treatments

$\begin{array}{ll}\mathrm{T}_{1}: & \mathrm{MS}+0.5 \mathrm{mg} \mathrm{l}^{-1} \mathrm{BAP}+0.1 \mathrm{mg} \mathrm{l}^{-1} \mathrm{NAA} \\ \mathrm{T}_{2}: & \mathrm{MS}+1.0 \mathrm{mg} \mathrm{l}^{-1} \mathrm{BAP}+0.1 \mathrm{mg} \mathrm{l}^{-1} \mathrm{NAA} \\ \mathrm{T}_{3}: & \mathrm{MS}+2.0 \mathrm{mg} \mathrm{l}^{-1} \mathrm{BAP}+0.1 \mathrm{mg} \mathrm{l}^{-1} \mathrm{NAA} \\ \mathrm{T}_{4}: & \mathrm{MS}+3.0 \mathrm{mg} \mathrm{l}^{-1} \mathrm{BAP}+0.1 \mathrm{mg} \mathrm{l}^{-1} \mathrm{NAA} \\ \mathrm{T}_{5}: & \mathrm{MS}+0.5 \mathrm{mg} \mathrm{l}^{-1} \mathrm{TDZ} \\ \mathrm{T}_{6} & \text { MS + } 1.0 \mathrm{mg} \mathrm{l}^{-1} \mathrm{TDZ} \\ \mathrm{T}_{7} & \text { MS medium devoid of any growth } \\ & \text { regulators as Control }\end{array}$


Fig.3.2 Shoot establishment of coccinea nodal segments in vitro as influenced by different growth regulator concentrations
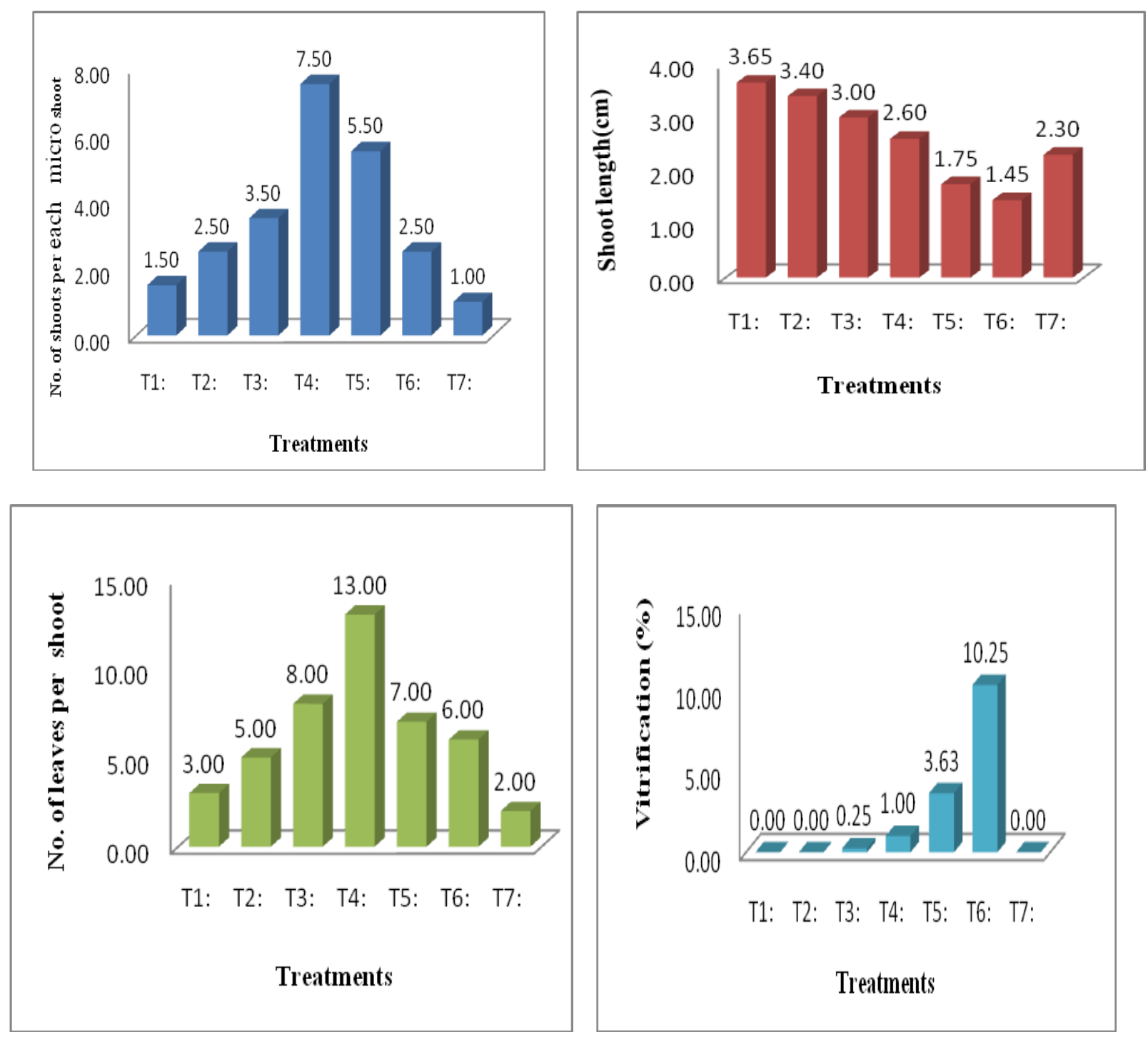

\section{Treatments}

$\mathrm{T}_{1}: \quad \mathrm{MS}+0.5 \mathrm{mg} \mathrm{l}^{-1} \mathrm{BAP}+0.25 \mathrm{mg} \mathrm{l}^{-1} \mathrm{NAA}$

$\mathrm{T}_{2}: \quad \mathrm{MS}+1.0 \mathrm{mg} \mathrm{l}^{-1} \mathrm{BAP}+0.25 \mathrm{mg} \mathrm{l}^{-1} \mathrm{NAA}$

$\mathrm{T}_{3}: \quad \mathrm{MS}+2.0 \mathrm{mg} \mathrm{l}^{-1} \mathrm{BAP}+0.25 \mathrm{mg} \mathrm{l}^{-1} \mathrm{NAA}$

$\mathrm{T}_{4}: \quad \mathrm{MS}+3.0 \mathrm{mg} \mathrm{l}^{-1} \mathrm{BAP}+0.25 \mathrm{mg} \mathrm{l}^{-1} \mathrm{NAA}$

$\mathrm{T}_{5}: \quad \mathrm{MS}+4.0 \mathrm{mg} \mathrm{l}^{-1} \mathrm{BAP}+0.25 \mathrm{mg} \mathrm{l}^{-1} \mathrm{NAA}$

$\mathrm{T}_{6} \quad \mathrm{MS}+5.0 \mathrm{mg} \mathrm{l}^{-1} \mathrm{BAP}+0.25 \mathrm{mg} \mathrm{l}^{-1} \mathrm{NAA}$

$\mathrm{T}_{7} \quad$ MS medium devoid of any growth regulators as control 
Plate.3.1 Culture establishment at 15 days after culture initiation
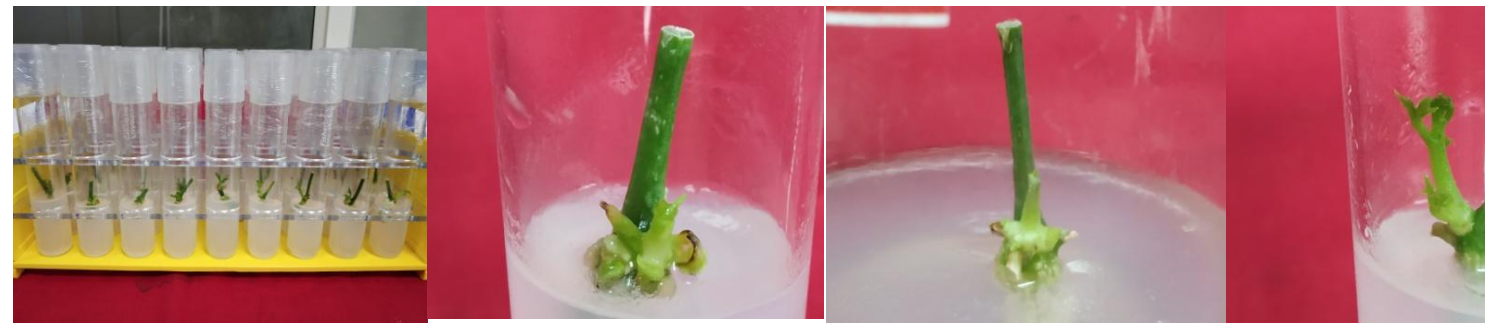

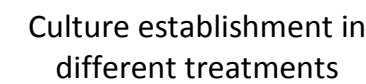

$0.5 \mathrm{BAP}+0.25$ NAA media

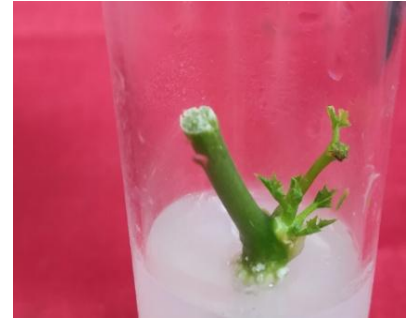

3.0 BAP + 0.25 NAA media
Bud sprouting in $2.0 \mathrm{mg} \mathrm{I}^{-}$ ${ }^{1}$ BAP
1.0 BAP + 0.25 NAA media

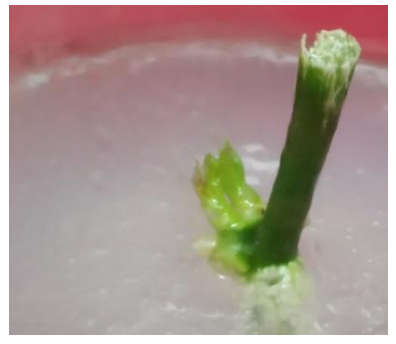

Bud sprouting in control
$2.0 \mathrm{BAP}+0.25 \mathrm{~N} /$

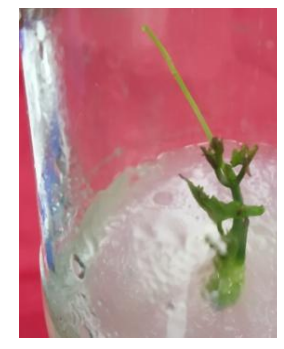

Shoot growth in (15 days after initiatior

Plate.3.2 Bud initiation and bud break for shoot production under in vitro shoot proliferation of coccinia nodal segments

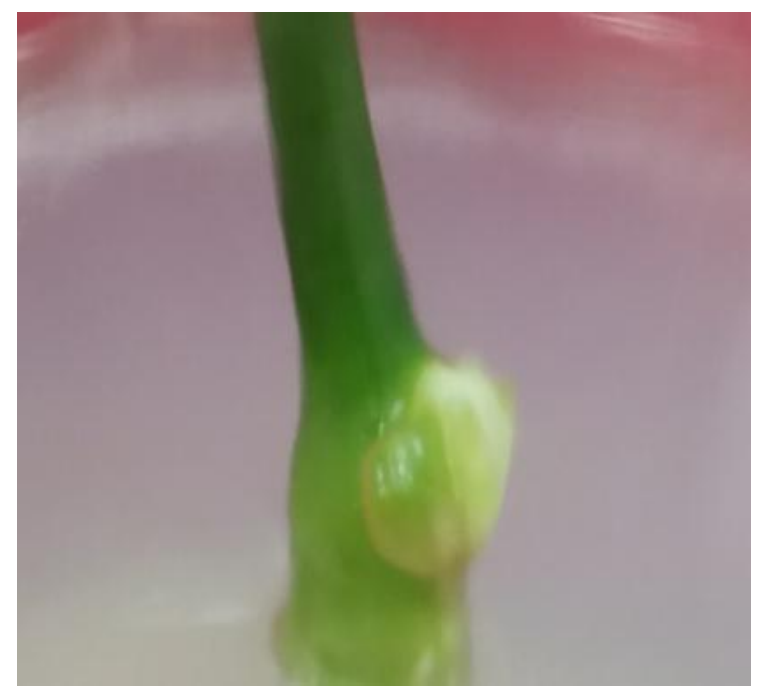

Swollen bud in culture initiation

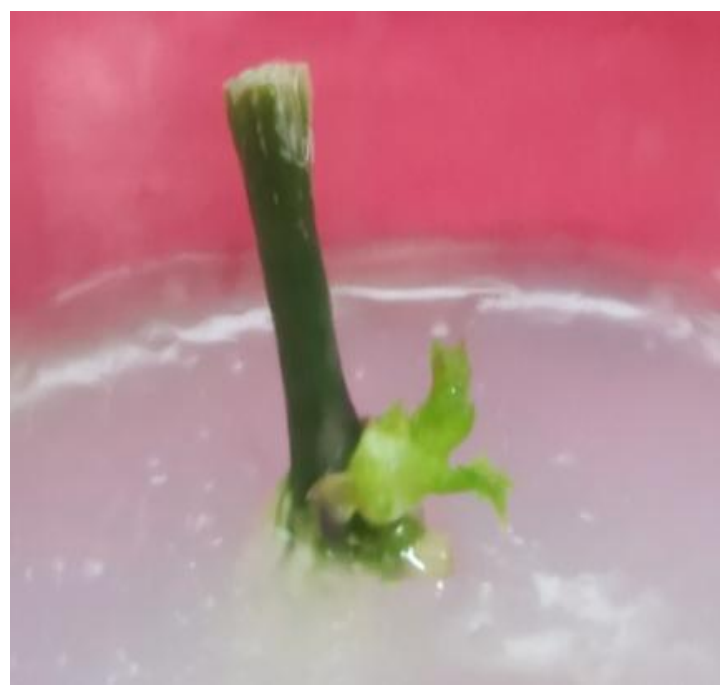

Bud break and shoot induction 
Plate.3.3 Effect of different growth regulators on Coccinia shoot proliferation15 days after treatment

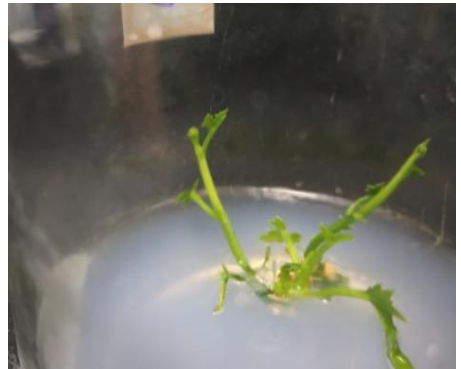

1.0 BAP + 0.25 NAA media

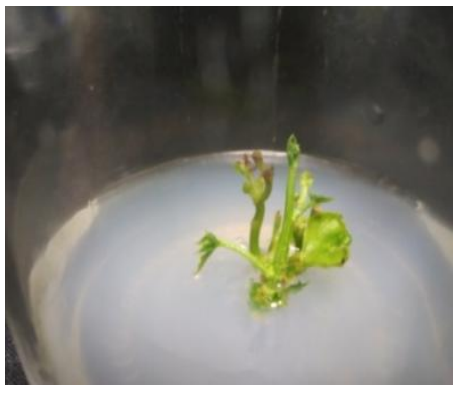

4.0 BAP + 0.25 NAA media

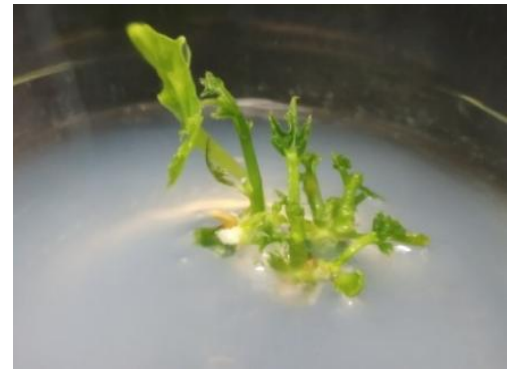

2.0 BAP + 0.25 NAA media

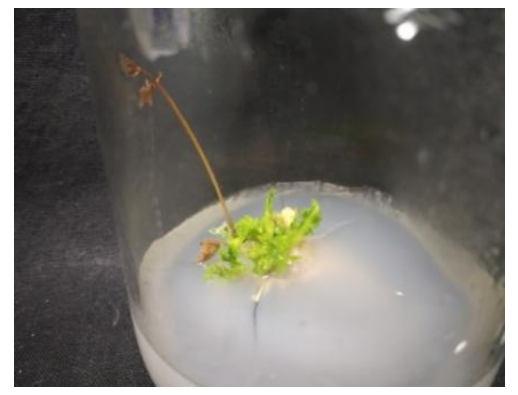

5.0 BAP + 0.25 NAA media

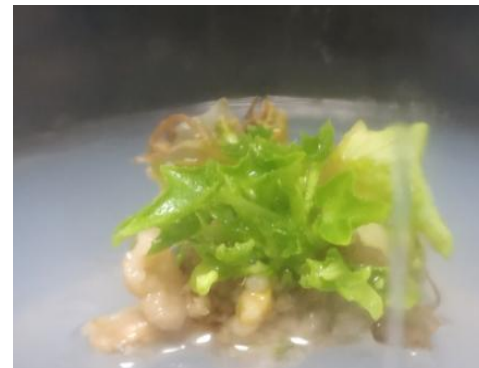

3.0 BAP + 0.25 NAA media (Best treatment)

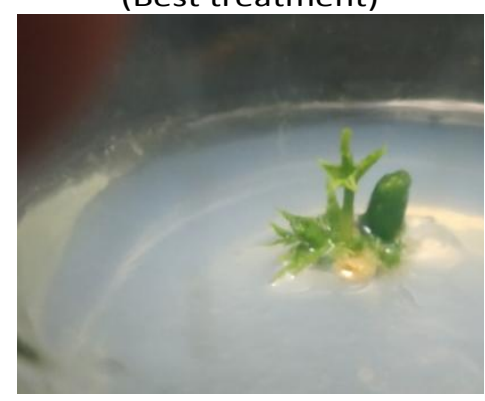

Control (devoid of growth regulators)

Plate.3.4 Bud initiation and bud break for shoot production under in vitro shoot proliferation of coccinia nodal segments

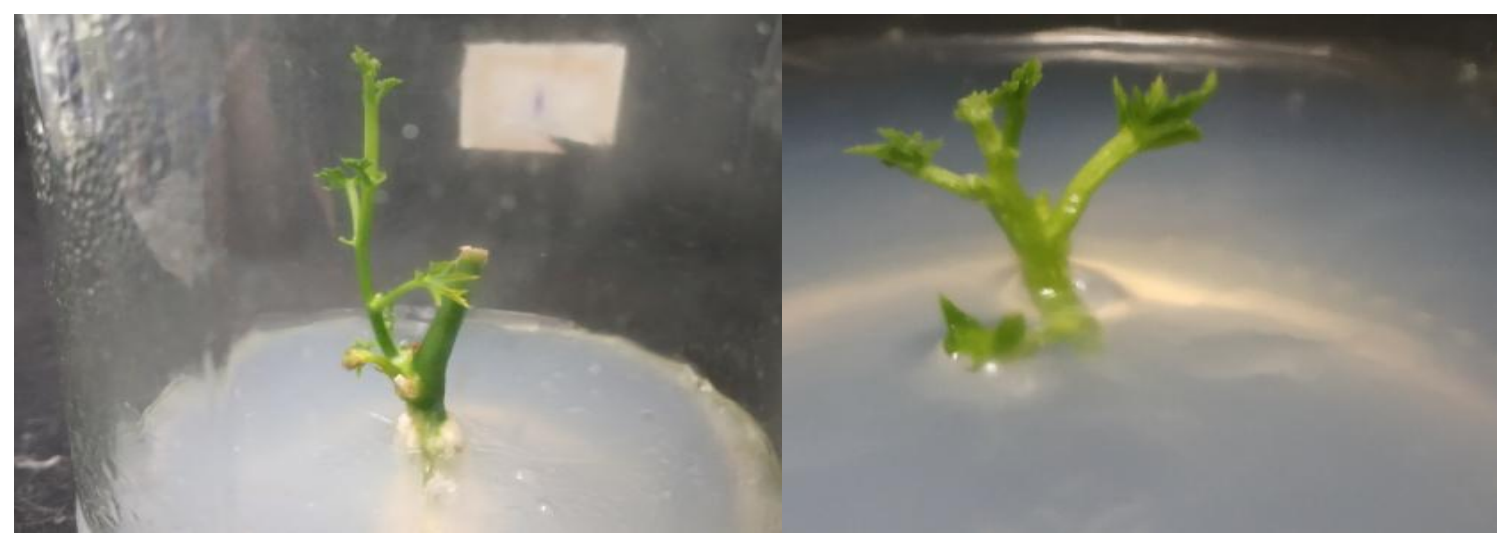

Well developed micro-shoot in culture establishment
Micro shoot cultured for shoot proliferation 


\section{Shoot proliferation}

Number of shoots per each micro shoot 30 days after inoculation

There were significant differences in respect of number of shoots per each micro shoot among the treatments. The mean number of shoots per each micro shoot 25 days after inoculation was 3.43 (Table 3.4). The highest number of shoots per each micro shoot (7.50) was observed by the MS + 3.0 $\mathrm{mg} \mathrm{l}^{-1} \mathrm{BAP}+$ $0.25 \mathrm{mg} \mathrm{l}^{-1} \mathrm{NAA}\left(\mathrm{T}_{4}\right)$ followed by $\mathrm{MS}+4.0$ $\mathrm{mg} \mathrm{l}^{-1} \mathrm{BAP}+0.25 \mathrm{mg}^{-1} \mathrm{NAA}\left(\mathrm{T}_{5}\right)(5.50)$ but significantly superior to the MS $+2.0 \mathrm{mg} \mathrm{l}^{-1}$ $\mathrm{BAP}+0.25 \mathrm{mg} \mathrm{l}^{-1} \mathrm{NAA}\left(\mathrm{T}_{3}\right)$ (3.50). However, the less number of shoots per each micro shoot was recorded in control $\left(\mathrm{T}_{7}\right)$ (1.00).

\section{Shoot length 30 days after inoculation}

Significant variation was observed among the treatments with respect to shoot length 30 days after inoculation. The mean shoot length was $2.59 \mathrm{~cm}$ (Table 3.4). The maximum shoot length was observed in MS $+0.5 \mathrm{mg} \mathrm{l}^{-1} \mathrm{BAP}$ $+0.25 \mathrm{mg} \mathrm{l}^{-1} \mathrm{NAA}\left(\mathrm{T}_{1}\right)(3.65 \mathrm{~cm})$ which was on par with MS + $1.0 \mathrm{mg} \mathrm{l}^{-1} \mathrm{BAP}+0.25 \mathrm{mg} \mathrm{l}^{-}$ ${ }^{1} \mathrm{NAA}\left(\mathrm{T}_{2}\right)(3.40 \mathrm{~cm})$ and $\mathrm{MS}+2.0 \mathrm{mg} \mathrm{l}^{-1}$ $\mathrm{BAP}+0.25 \mathrm{mg} \mathrm{l}^{-1} \mathrm{NAA}\left(\mathrm{T}_{3}\right)(3.00 \mathrm{~cm})$ but significantly superior to $\mathrm{MS}+3.0 \mathrm{mg} \mathrm{l}^{-1} \mathrm{BAP}$ $+0.25 \mathrm{mg} \mathrm{l}^{-1} \mathrm{NAA}\left(\mathrm{T}_{4}\right)(2.60 \mathrm{~cm})$. However, the minimum shoot length was recorded in MS medium devoid of any growth regulators as control $(1.75 \mathrm{~cm})$.

\section{Number of leaves per shoot}

The data revealed that there were significant differences among the treatments in respect of number of leaves per shoot. The mean of number of leaves per shoot was 6.29 (Table 3.5). The maximum number of leaves per shoot (13.0) was exhibited in MS + $3.0 \mathrm{mg} \mathrm{l}^{-1}$ $\mathrm{BAP}+0.25 \mathrm{mg} \mathrm{l}^{-1} \mathrm{NAA}\left(\mathrm{T}_{4}\right)$ followed by MS $+2.0 \mathrm{mg} \mathrm{l}^{-1} \mathrm{BAP}+0.25 \mathrm{mg} \mathrm{l}^{-1} \mathrm{NAA}\left(\mathrm{T}_{3}\right)$
(8.0) but significantly superior to the MS medium devoid of any growth regulators as control $\left(T_{5}\right)$ (7.00). However, the minimum number of leaves per shoot was observed in control (2.00).

\section{Leaf area $\left(\mathrm{cm}^{2}\right)\left(3^{\text {rd }}\right.$ leaf from top $)$}

Significant differences were observed among the treatments with respect to leaf area. The mean leaf area was $4.24 \mathrm{~cm} 2$ (Table 3.5). The maximum leaf area was observed in MS + 0.5 $\mathrm{mg} \mathrm{l}^{-1} \mathrm{BAP}+0.25 \mathrm{mg} \mathrm{l}^{-1} \mathrm{NAA}\left(\mathrm{T}_{1}\right)(6.22$ $\mathrm{cm} 2$ ) which was on par with $\mathrm{MS}+1.0 \mathrm{mg}^{-1}$ $\mathrm{BAP}+0.25 \mathrm{mg} \mathrm{l}^{-1} \mathrm{NAA}\left(\mathrm{T}_{2}\right)(5.60 \mathrm{~cm} 2)$ but significantly superior to the MS medium devoid of any growth regulators as

Control $\left(\mathrm{T}_{5}\right) \quad\left(5.00 \mathrm{~cm}^{2}\right)$. However, the minimum leaf area was recorded in $\mathrm{MS}+3.0$ $\mathrm{mg} \mathrm{l}^{-1} \mathrm{BAP}+0.25 \mathrm{mg}^{-1} \mathrm{NAA}\left(\mathrm{T}_{2}\right)(2.10$ $\mathrm{cm}^{2}$ ).

\section{Vitrification (\%)}

Differences among the treatments were found to be significant for vitrification. The mean per cent of vitrification was $2.16 \%$ (Table 3.5). The highest per cent of vitrification $(10.25 \%)$ was observed by the MS + $3.0 \mathrm{mg} \mathrm{l}^{-}$ ${ }^{1} \mathrm{BAP}+0.25 \mathrm{mg} \mathrm{l}^{-1} \mathrm{NAA}\left(\mathrm{T}_{4}\right)$ followed by $\mathrm{MS}+4.0 \mathrm{mg} \mathrm{l}^{-1} \mathrm{BAP}+0.25 \mathrm{mg} \mathrm{l}^{-1} \mathrm{NAA}\left(\mathrm{T}_{3}\right)$ $(3.62 \%)$. The least per cent of vitrification $(0.00 \%)$ was recorded in MS medium devoid of any growth regulators as control $\left(\mathrm{T}_{7}\right)$, MS $+0.5 \mathrm{mg} \mathrm{l}^{-1} \mathrm{BAP}+0.25 \mathrm{mg} \mathrm{l}^{-1} \mathrm{NAA}\left(\mathrm{T}_{1}\right)$ and $\mathrm{MS}+1.0 \mathrm{mg} \mathrm{l}^{-1} \mathrm{BAP}+0.25 \mathrm{mg} \mathrm{l}^{-1} \mathrm{NAA}\left(\mathrm{T}_{2}\right)$.

It is evident from the above results on shoot proliferation study that the $3.0 \mathrm{mg} \mathrm{l}^{-1} \mathrm{BAP}+$ $0.25 \mathrm{mg} \mathrm{l}^{-1} \mathrm{NAA}\left(\mathrm{T}_{4}\right)$ and $4.0 \mathrm{mg} \mathrm{l}^{-1} \mathrm{BAP}+$ $0.25 \mathrm{mg} \mathrm{l}^{-1} \mathrm{NAA}\left(\mathrm{T}_{5}\right)$ in MS medium were more efficient for inducing maximum number of micro shoots and leaves over it. On the contrary, the shoot growth and leaf growth were at maximum levels by the use of $0.5 \mathrm{mg}$ 
$\mathrm{l}^{-1} \mathrm{BAP}+0.25 \mathrm{mg} \mathrm{l}^{-1} \mathrm{NAA}\left(\mathrm{T}_{1}\right)$ on par with $\mathrm{MS}+1.0 \mathrm{mg} \mathrm{l}^{-1} \mathrm{BAP}+0.25 \mathrm{mg} \mathrm{l}^{-1} \mathrm{NAA}\left(\mathrm{T}_{2}\right)$. Those concentrations amounting to higher levels of multiple shoot induction with high vitrification lagged behind in growth. Therefore, it can be summarised that a moderate performance in both multiplicity of induction and eventual growth could be achieved by $1.0 \mathrm{mg} \mathrm{l}^{-1} \mathrm{BAP}+0.25 \mathrm{mg} \mathrm{l}^{-1}$ $\mathrm{NAA}\left(\mathrm{T}_{2}\right)$ and $2.0 \mathrm{mg} \mathrm{l}^{-1} \mathrm{BAP}+0.25 \mathrm{mg} \mathrm{l}^{-1}$ NAA $\left(T_{3}\right)$. However, those treatments which were less efficient in the shoot induction and eventual growth of both shoot and leaf in sub cultures required extended duration to advance the cycles.

Media with the higher cytokinin concentration showed the maximum number of multiple shoots may be due to the fact that suppression of apical dominance leads to the production of more number of multiple shoots and reduced shoot length (Balachandran et al., (1990) and Ali et al., (2004)).

Kurmi et al., (2011) stated that TDZ was found more effective at lower concentrations $\left(0.1-0.5 \mathrm{mg} \mathrm{l}^{-1}\right)$. TDZ also affected auxin transport in hypocotyl tissues of Pelargonium and others (Murch and Saxena, 2001) and promoted regeneration frequency by altering the levels of abscisic acid, ethylene and perhaps proline (Murch and Saxena, 2001).

\section{References}

Aasim, M, Khawar KM, Ozcan S. 2008. In vitro micropropagation from shoot meristems of Turkish cowpea (Vigna unguiculata L.) cv. Akkiz. Bangladesh Journal of Botany. 37(2):149-54.

Ali, A, Munawar, A. and Siddiqui, F.A.2004. In vitro propagation of turmeric Curcumin longa L. International Journal of Biology and Biotechnology. 1: 511-18.

Aragaw, Z and Abebe, W. 2017. Protocol optimization for micro-propagation of Green pepper (Capsicum annum L.) cultivated in Ethiopia. Journal of Medicinal Plants Studies. 6(1): 22934.

Arulanandam, L.J.P. and S. Ghanthikumar, 2011. Short communications: Indirect organogenesis of Vitex trifolia L-An important medicinal plant. Indian Journal of Natural Products and Resources. 2: 261-64.

Balachandran SE, Osmond CB, Robinson, Slims DA. 1990. Concepts of plant biotic stress. Some insights into the stress physiology of virus- infected plants, from the perspective of photosynthesis. Physiologia Plantarum. 100:203-13.

Feng, J.H. and J.T. Chen. 2014. A novel in vitro protocol for inducing direct somatic embryogenesis in Phalaenopsis aphrodite without taking explants. Scientific World Journal.17.

Hasan, M.F, Jannat, M.M, Zaman, R. and Sikdar, B. 2019. Virus-free plant regeneration from shoot apical meristem of Coccinia grandis L. an important medicinal plant in Bangladesh. Recent Advances in Biology and Medicine. 5:1-7.

Huetteman, C.A. 1993. In vitro culture of Juglans nigra L.: Micropropagation from embryonic axes and forcing of adult quiescent stems. M.S. Thesis. SouthernIllinoisUniversity,Carbondale.

Kahia, J, Njenga, P and Kirika, M. 2016. Improved Micropropagation of Plantlets from Nodal Explants of Anchote (Coccinia abyssinica)-A Calcium- and Protein-rich Tuber. Hort Science. 51(7):905-09.

Khawar, K.M Sancak,, C, Uranbey, S. and Ozcan, S. 2004. Effect of Thidiazuron on shoot regeneration from different 
explants of lentil (Lens culinaris Medik.) via organogenesis. Turkey Journal of Botany. 28: 421-26.

Kurmi, U.S, Sharma, D.K, Tripathi, M.K, Tiwari, R, Baghel, B.S. and Tiwari, S. 2011. Plant regeneration of Vitis vinifera (L) via direct and indirect organogenesis from cultured nodal segments. Journal of Agricultural Technology. 7(3): 721-37.

Mok, M. C. and Mok, W.S. 1987. Biological and biochemical effects of cytokinin active phenylurea derivates in tissue culture systems. Hort Science. 26:1194-97.

Mukund, T. 1998. Standardization of micropropagation techniques banana cv. Kadali (Musa acuminate Colla). M. Sc. (Agri.) Thesis, University of Agricultural Sciences, Bangalore, Karnataka.

Murch, S. J.and Saxena, P.K. 2001. Molecular fate of thidizuron and its effects on auxin transport in hypocotyls tissues of Pelargonium x hortorum Bailey. Journal of Plant Growth Regulation.35:269-75.

Senthilkumar, P, Paulsamy, S, Vijayakumar, K. K. and Kalimuthu, K. 2007. In vitro regeneration of the medicinal herbs of Nilgiri Shola, Acmella calva L. from leaf derived callus. Plant Tissue Culture and Biotechnology, 17(2): 109-14.

Shekhawat, M.S, Ravindran, C. P and Manokari, M. 2014. Developmental and Hormonal Regulation of direct shoots and roots regeneration in Coccinia indica. International Journal of Natural Sciences Research, 2(7): 103-12.

Ugandhar T, Devi A, Srilatha T, SammaiahD. 2015. Plant Let Regeneration from Leaf Explants through Organogenesis in Bitter Melon (Momordica charantia L.). Academic Journal of Interdisciplinary Studies. 3:79-84.

\section{How to cite this article:}

Mohammad Amin, A. V. D. Dorajee Rao, K. Ravindra Kumar and Salomi Suneetha, D. R. 2020. Culture Establishment of Coccinia grandis (L.) Through Nodal Segments under in vitro Conditions. Int.J.Curr.Microbiol.App.Sci. 9(07): 2273-2286.

doi: https://doi.org/10.20546/ijcmas.2020.907.265 